\title{
TURBULENCE ASPECTS OF MASS TRANSFER IN THE THIN INTERFACIAL REGION OF THE CONCENTRATION BOUNDARY LAYER IN GAS-LIQUID SYSTEMS
}

\author{
HARRY EDMAR SCHULZ ${ }^{1}$, FRANCISCO ANTONIO LOYOLA LAVIN ${ }^{2} \&$ \\ BRUNO BATISTA GONÇALVES ${ }^{3}$ \\ ${ }^{1}$ School of Engineering at São Carlos, University of São Paulo, Brazil. \\ ${ }^{2}$ Faculty of Engineering, University of Magallanes, Chile. \\ ${ }^{3}$ University Centre of Patos de Minas, Brazil.
}

\begin{abstract}
The quantification of overall mass transfers in gas-liquid systems depends on the spatial evolution of the relevant variables close to the interface of the two phases. When turbulence is present (in the present study the turbulence is considered in the liquid phase), the methods of treating the problem consider the differential form of the momentum and mass conservation equations. The continuous hypothesis that underlies these equations in principle allows verifying the limiting trends very close to the interface. Because the theoretical concepts of turbulence are defined using statistical tools, the mentioned verification depends on the intrinsic definitions used in the statistical approach. In this study the turbulent mass transfer parameters are calculated for the thin region close to the interface based on the tool of random square waves (RSW). Theoretical results are obtained and analyzed in the context of existing experimental data and conceptual discussions of the literature, using a constant 'reduction function', a parameter defined in this methodology. The results of the present analysis show that the RSW method allows obtaining functional trends, as well as indicate the adequacy of using a variable reduction function to better represent reality.

Keywords: concentration fluctuations, gas-liquid interfaces, RSW, turbulence statistics.
\end{abstract}

\section{INTRODUCTION}

Transfer phenomena related to moving interfaces, like the gas liquid interfaces, are still not definitively quantified, a situation more evident if turbulence is present. From the molecular point of view, the interface is a transition region from the gaseous phase to the liquid phase, having a thickness of several molecular diameters of the species that compose the system. From the macroscopic point of view, the interface is a continuous surface, and the physical properties of the species under study are defined at this surface applying thermodynamic or constitutive laws. Turbulence must be taken into account in both cases: (1) it is a macroscopic 'random' event composed by 'immense' populations of molecules that interferes in the molecular interaction of the volumetric interfacial region, or (2) it is a continuous 'random' event that interacts with the defined surface imposing local (microscopic) conditions for the transfer processes to occur. The term 'random' suggests the use of statistical tools in both approaches. The second approach, macroscopic and continuous, is the one considered in the present study.

The liquid side of the turbulent air-water interface is traditionally viewed as a superposition of sublayers that coexist in the region that forms the 'surface-induced layer' [1]. Figure 1 shows the idealized structure. The thickness ' $S$ ' of this layer was defined as the far integral length scale $L_{\infty}$, considering that larger distances would not depend on viscous effects. The thickness of the so-called 'viscous sublayer' is indicated by ' $V$ ', and smaller distances to the surface are affected by viscous effects [2]. The turbulent Reynolds number $\mathrm{Re}_{\mathrm{t}}$ is used to quantify a mean value for $V$. Considering the Definition of Kolmogorov for the smallest eddies in 


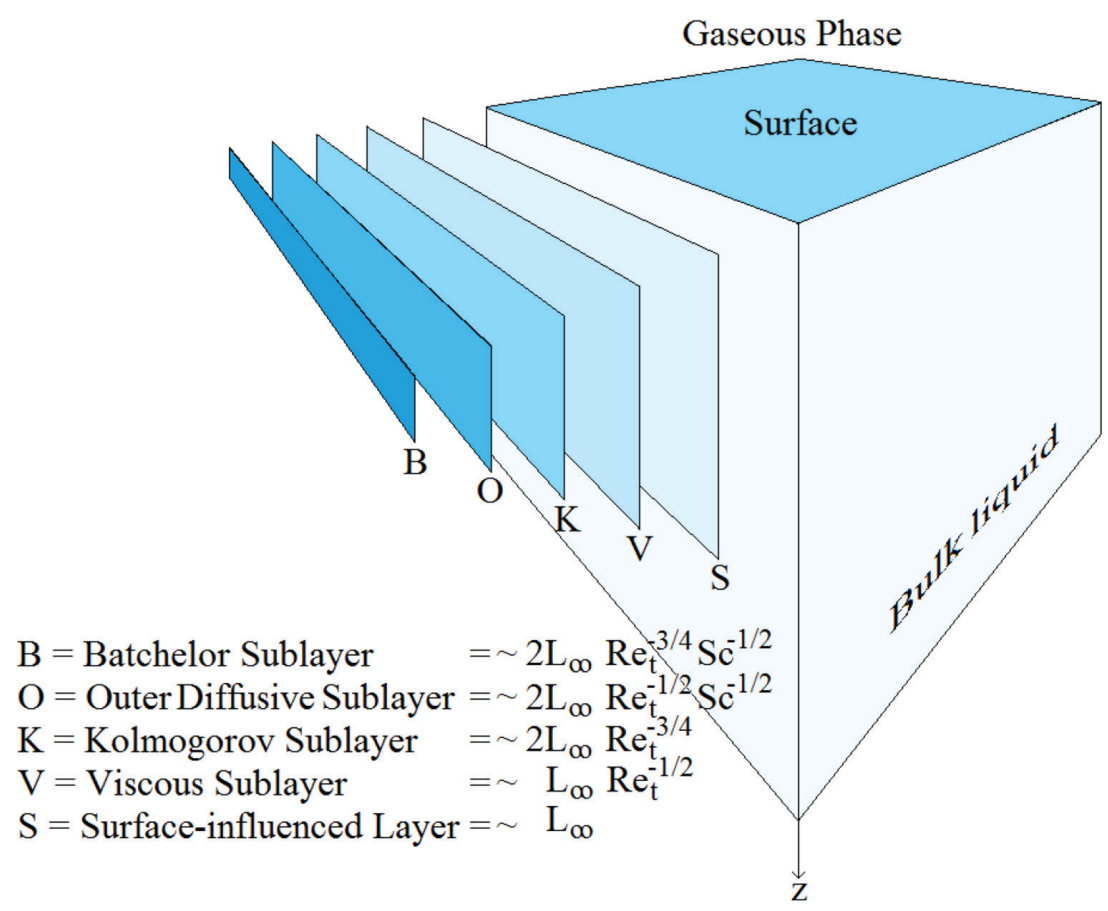

Figure 1: Superficial structure of successive sublayers that coexist in the region influenced by the surface during mass transfer.

isotropic turbulence, the thickness ' $K$ ' is defined for the 'Kolmogorov sublayer' using the same variables of the viscous sub-layer. When mass transfer occurs, an 'outer-diffusive sublayer' is defined, which inserts the Schmidt number Sc in the quantification of the mean value of the thickness ' $O$ '. Finally, very close to the surface, the 'Batchelor sublayer' is defined with thickness ' $B$ ', representing the mean distance that is more affected by characteristics of the smallest eddies in mass transfer. This last sublayer uses the same parameters of the outer-diffusive sublayer for its quantification. In the sketch of Fig. 1, it is assumed that $\mathrm{Sc}>1$.

Measurements of independent and of simultaneous instantaneous concentration and velocity fields close to the air-water interface may be found in the literature, as Refs. [3-11], for example. These measurements allow obtaining statistical parameters like mean concentration profiles, concentration RMS profiles and experimental estimates of turbulent mass fluxes. When describing the used experimental optical techniques, different authors mention adjustments of the concentration measurements in the very near surface region, and interpolations for the velocity data, which have influences in the evaluation of the turbulent mass fluxes, for example, [4, 6-8]. Figure 2 illustrates schematically the obtainment of mean concentration fields from instantaneous data, together with a sketch of the thickness of the sublayers of Fig. 1, emphasizing its sense as mean values. Details of the instantaneous concentration fields may be found in Refs. [3] and [4].

\section{MATHEMATICAL MODELS}

In the method of random square waves (RSW) variables that oscillate between two limiting values (boundary layer like problems) have their statistical properties evaluated using 


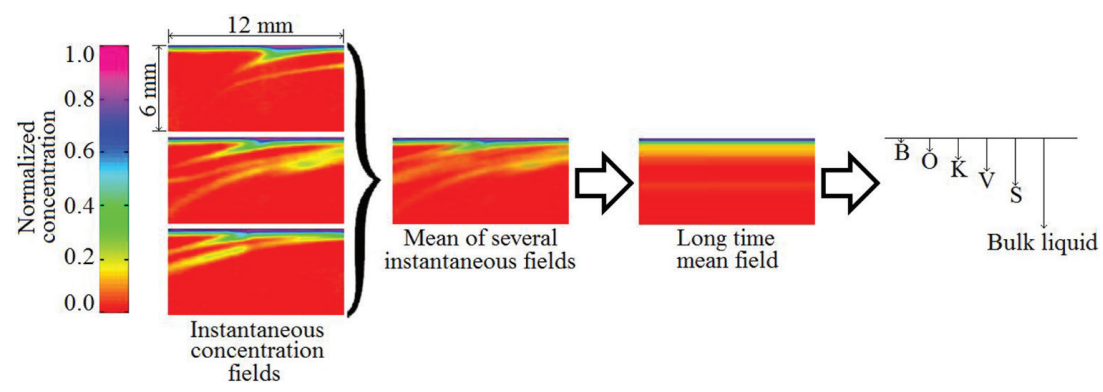

Figure 2: Mean statistical properties obtained from series of instantaneous measured fields.

bi-modal random signals and corrective functions. In the 1D case of scalar transport four such functions are needed. Thus, as mentioned by Refs. [12] and [13], also four equations are needed. By rearranging terms, two of the parameters were linked, remaining three to be solved through only three equations. By considering one of the parameters constant (the reduction function ' $\alpha$ ') it was possible to reduce the problem substantially and establish one equation for the so-called partition function ' $n$ '. In fact, the reduction function varies with $z$, the distance to the surface, and profiles of ' $\alpha$ ' obtained from experimental data may be found in Refs. [14] and [8], for example (see Fig. 3). For stationary turbulence and constant $\alpha$ the equation for $n$ was theoretically solved ([15] and [16]), allowing checking the behavior of the mean concentration profile. Equations (1) and (2) are the 1D mass conservation equation and the nondimensional form of the turbulent mas flux for the RSW method, respectively.

$$
\begin{gathered}
0=\frac{d^{2} n}{d z^{2}}-\frac{d I J *}{d z^{*}}, z^{*}=\frac{z}{E}, \\
I^{*}=\frac{n(1-n) A\left(E \sqrt{\left.\overline{\omega^{2}} / D\right)}\right.}{\sqrt{n(1-n)+\frac{\beta(1-\beta)}{(2 \beta-1)^{2}}}}=\frac{n(1-n) A \sqrt{\overline{\omega^{* 2}}}}{\sqrt{n(1-n)+\frac{\beta(1-\beta)}{(2 \beta-1)^{2}}}}=\overline{\omega^{*} c^{*}}, c^{*}=\frac{c}{C_{S}-C_{b}} .
\end{gathered}
$$

These equations show the four mentioned functions: $n$, the cited 'partition function', which coincides with the normalized mean concentration; $\beta$ is the normalized 'superposition function', which quantifies the coherence between concentration and velocity fluctuations; $\sqrt{\overline{\omega^{2}}}$ is the RMS of $\omega$, the fluctuation of the vertical velocity $\left(\mathrm{ms}^{-1}\right)$, being $\sqrt{\overline{\omega^{*^{2}}}}$ the normalized intensity of turbulence and $A=1-\alpha$ replaces the normalized reduction function. $c^{*}$ and $z^{*}$ are the normalized concentration fluctuation and the vertical dimension, respectively, the last with origin at the surface and positive downwards; $D$ is the molecular diffusivity of the gas in the liquid $\left(\mathrm{ms}^{-2}\right), E$ is the concentration boundary layer thickness $(\mathrm{m})$, given by the position where $n=0.01 ; I J^{*}=\omega^{*} c^{*}$ is the normalized turbulent mass flux. $C_{S}$ and $C_{b}$ are the concentrations of gas at the surface and the bulk liquid, respectively $\left(\mathrm{kg} \cdot \mathrm{m}^{-3}\right)$. The integration of eqn (1) furnishes the mass flux that crosses the medium, $j^{*}=-d n / d z^{*}+I J^{*}$, nondimensional and constant along $z^{*}$ (integration constant). Equation (1) shows that if $d n / d z^{*}$ has a critical point (maximum or minimum) in the physical domain, then $I J^{*}$ also has a critical point at the same position. By assuming a minimum value of zero for $I J^{*}$, the integration constant assumes the value $j^{*}=-d n / d z^{*}{ }_{\text {critical }}$. On the other hand, considering the structure shown in Figs 1 and 2, 
where diffusion is dominant at the surface, and for which no vertical movement was proposed, $\sqrt{\overline{\omega^{* 2}}}=0$ at $z^{*}=0$ may be used, implying $I J^{*}=0$, and $j^{*}=-d n /\left.d z^{*}\right|_{z^{*}=0}$. This situation can also be applied when $d n / d z^{*}$ has no critical point. For the two described situations we have, then:

$$
I J^{*}=\frac{d n}{d z^{*}}-\left.\frac{d n}{d z^{*}}\right|_{\text {critical }} \text { and } I J^{*}=\frac{d n}{d z^{*}}-\left.\frac{d n}{d z^{*}}\right|_{z^{*}=0}
$$

2.1 Literature Solutions for ' $n$ '

The equation furnished by Ref. [16] for the mean concentration $n$ and any constant value of $A=1-\alpha$ is:

$$
\frac{d n}{d z^{*}}=K\left(\frac{1+\theta_{2}}{1-\theta_{2}}\right)^{\theta_{1} / 2}\left[\left(\frac{1-\frac{\theta_{3} n}{1+\theta_{2}}}{1+\frac{\theta_{3} n}{1-\theta_{2}}}\right)^{\theta_{1} / 2}-1\right], \quad\left\{\begin{array}{l}
\theta_{1}=2(1-A) /\left[A \sqrt{4 A^{2}+1}\right] \\
\theta_{2}=(2 A-1) / \sqrt{4 A^{2}+1} \\
\theta_{3}=4 A / \sqrt{4 A^{2}+1}
\end{array} .\right.
$$

$K$ is a nondimensional constant coefficient. Equation (4) was integrated for several values of $\theta_{1}$, or $A$, the last being a measure of the relative influence between molecular diffusivity and turbulence in the transfer phenomenon, or, equivalently, a measure of agitation. In the present study the values $\theta_{1}=2$ (or $A=0.43099$ ) and $\theta_{1}=4$ (or $A=0.30007$ ) are considered, which allow integrating eqn (4) obtaining eqns (5a) and (5b), respectively:

$$
\begin{gathered}
z^{*}=-0.20470[n+0.84587 \ln (n)-1] \\
z^{*}=-0.19690[0.68076 \ln (n)-5.7913 \ln (2.5009-n)-n+3.3517] .
\end{gathered}
$$

Figure 3 allows visualizing the assumption of constant ' $\alpha$ ' in the range of possible profiles of the reduction function. The grey cloud considers the sets of profiles shown in Refs. [8] and [14] for different agitation conditions in a tank with oscillating grids. The values of ' $\alpha$ ' used in eqns (5a) and (5b) are shown in the figure.

Figure 4 shows calculated $n$ profiles for different values of $\theta_{1}$, including $\theta_{1}=2$ (or $A=0.43099$ ) and $\theta_{1}=4$ (or $A=0.30007$ ). Data of Ref. [6], presented as a grey cloud, were

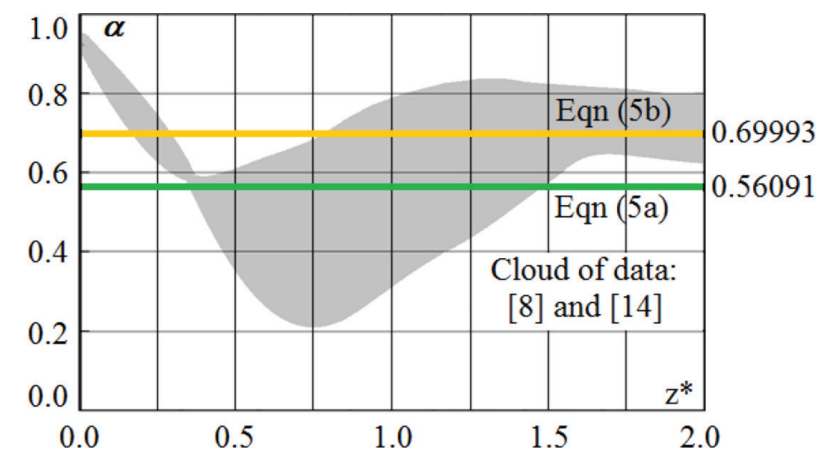

Figure 3: Experimental data of $\alpha$ varying along $z^{*}$, and the two constant values used here. 


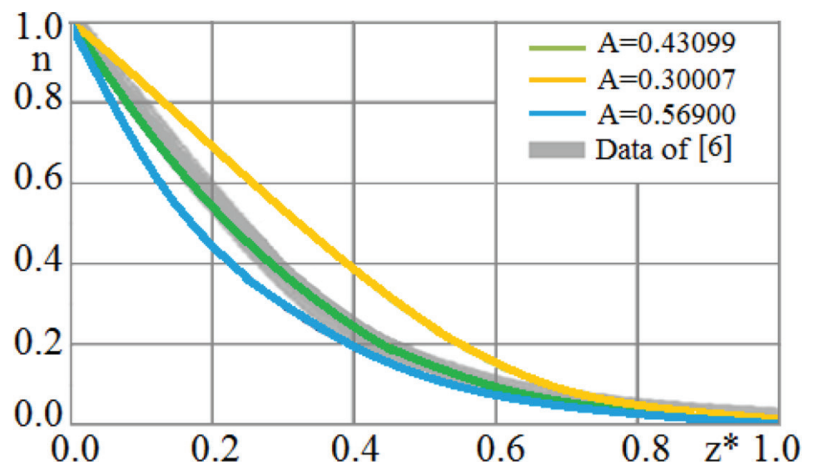

Figure 4: Theoretical $n$ profiles compared with experimental data.

compared with the calculated profiles, and the agreement with the solution obtained for $\theta_{1}=2$ is remarkable $([16,17])$.

\section{STATISTICAL FUNCTIONS FOR EQUATIONS (5A) AND (5B)}

\subsection{Central Moments: the Concentration RMS Function}

$n, \alpha, \beta$, and $\sqrt{\overline{\omega^{2}}}$ allow obtaining any statistical function related to mass transfer for the boundary layer situation under study. The normalized central moments of the concentration fluctuations need only $A$ and $n$, and are given by eqn (6) (see Ref. [12]), for $\eta=1,2,3 \ldots$ The RMS of the concentration fluctuation is obtained for $\eta=2$.

$$
\sqrt[\eta]{\overline{c^{* \eta}}}=\sqrt[\eta]{n(1-n)\left[(1-n)^{\eta-1}+(-1)^{\eta} n^{\eta-1}\right]} A
$$

Equations (5a) and (5b) and the correspondent moments obtained through eqn (6) are plotted together against $z^{*}$ in Fig. 5. Because all calculated profiles depend on $n$, Fig. 5a shows the profiles $n$ of eqns (5a) and (5b) and the RMS concentration, while Fig. 5b shows higher order moments.

The full lines of Fig. 5a were already presented in Fig. 4. Because $\theta_{1}$ (or $A$ ) are measures of the agitation, these curves show that different agitation conditions produce different normalized profiles. For $\eta=2$, eqn (6) furnishes the RMS function, given by:

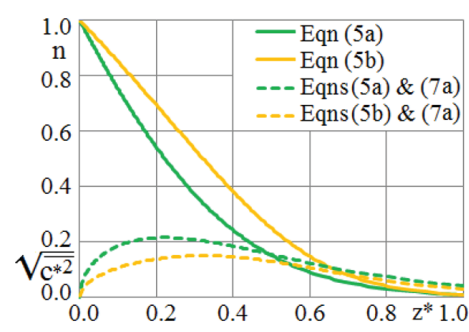

(a)

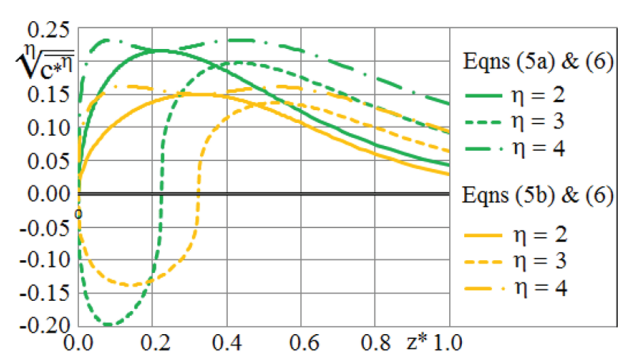

(b)

Figure 5: (a) $n$ and concentration RMS profiles; (b) Higher order central moment profiles. 


$$
\sqrt{\overline{c^{* 2}}}=\sqrt{n(1-n)} A
$$

Using eqn (5a) and assuming a power law $\overline{c^{* 2}}=c_{1} z^{* q}$ for $z^{* \rightarrow 0}$, or $n \rightarrow 1$, where $c_{1}$ is a constant, it is obtained that the RMS of the fluctuation increases at this position as

$$
\sqrt{\overline{c^{* 2}}}=\sim 0.70115 \sqrt{z^{*}} \text {. }
$$

Using eqn (5b) and steps similar to that followed to obtain eqn (7b), the result is:

$$
\sqrt{\overline{c^{* 2}}}=\sim 0.35945 \sqrt{z^{*}} \text {. }
$$

Equations (7a) and (7b) show that the solutions for constant ' $\alpha$ ' produce RMS profiles that are paralel to the $\sqrt[\eta]{\overline{c^{* \eta}}}$ axis at $z^{*}=0$. That is, the derivative of the RMS function tends to inifinite at this point. Figure 6 shows experimental and theoretical RMS profiles, the last obtained with eqns (5a), (5b) and (7). In this figure the horizontal axis follows, for convenience to reproduce the data, the normalization adopted by [5] as $z+=z / \delta$, being $\delta$ the position where the concentration difference $C(z)-C_{b}$ atains $36.7 \%$ of the total difference $C_{S}-C_{b}$. The shape of the theoretical solutions follow the experimental data. Maximum values and peak positions are in the range of the observed values, and eqn (5a) follows more closely the observed data. The

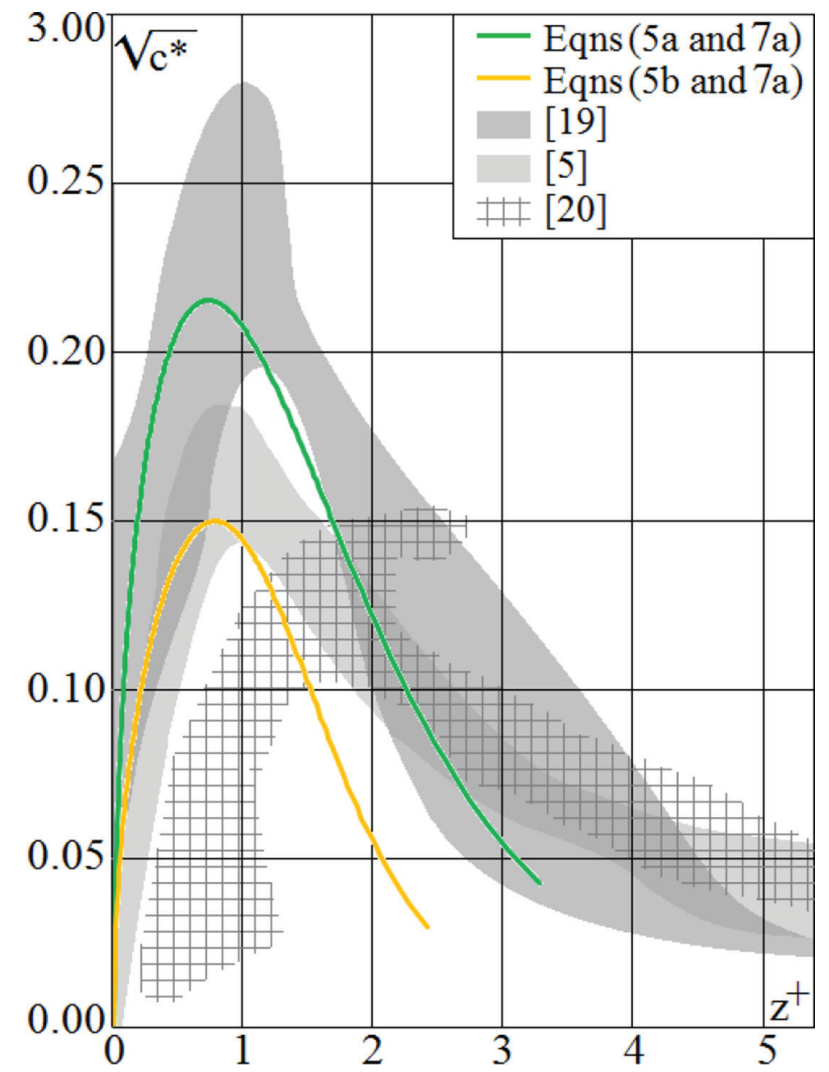

Figure 6: Experimental and theoretical concentration RMS profiles. Data of $[5,19,20]$. 
experiments represented by the light grey cloud (envelope of data of Ref. [5]) show a finite slope (derivative) of the RMS function at $z^{*}=0$. The studies of Refs. $[3,4,6,8,10]$ also show RMS profiles with well-defined finite slopes at the origin. The study of Ref. [18] shows RMS results that do not attain zero at $z^{*}=0$, although they also approach the surface with a finite slope. Such observed finite values imply that constant ' $\alpha$ ' solutions probably not describe properly concentration RMS details at the origin, for mass transfer descriptions. Figure 3 shows that $\alpha$ itself tends to zero at this point, and the maintenance of the nonzero constant value near the surface may be the cause of the observed differences between the theoretical and experimental RMS profiles. In general, as already mentioned, the theoretical solution for $\theta_{1}=2$, eqn (5a), represents better the experimental data.

\subsection{Covariances: the Turbulent Transport Function}

Although $\beta$ and $\omega$ are still unknown functions, they appear combined in $I J^{*}=\overline{\omega^{*} c^{*}}$, which, together with the mentioned solutions, allow obtaining a second set of relevant statistical functions and analysing them. Equations (3) show that $\omega^{*} c^{*}$ is related to the derivative of $n$ given by eqn (4). When using equations (5a) and (5b) the derivatives simplify, respectively, to:

$$
\begin{gathered}
d n / d z^{*}=-4.8853 n /(n+0.84587) \\
d n / d z^{*}=-5.0787 n(2.5009-n) /(1.3048+n)^{2}
\end{gathered}
$$

Equation (8a) has no critical point for $0 \leq n \leq 1$. So, using eqns. (2), (3) and (8a), we have:

$$
\overline{\omega^{*} c^{*}}=-4.8853 n /(n+0.84587)+2.6466
$$

Equation (8b) has a critical point at $n=0.63852$. Using $\overline{\omega^{*} c^{*}}=0$ at this position, the equation for the turbulent flux is:

$$
\overline{\omega^{*} c^{*}}=-5.0787 n(2.5009-n) /(1.3048+n)^{2}+1.5992
$$

Alternatively, when using the condition $\sqrt{\overline{\omega^{*^{2}}}}=0$ at $z^{*}=0$, we have:

$$
\overline{\omega^{*} c^{*}}=-5.0787 n(2.5009-n) /(1.3048+n)^{2}+1.4350
$$

Equation (9a) produces positive $\overline{\omega^{*} c^{*}}$ for $z^{*}>0$, and a zero value at $z^{*}=0$. About eqns. (9b) and $(9 \mathrm{c})$, although the small difference between them, the profiles are qualitatively different, as shown in Fig. 7. Equation (9b) shows a positive function $\overline{\omega^{*} c^{*}}$ with a nonzero value at $z^{*}=0$, while eqn (9c) shows a negative $\omega^{*} c^{*}$ close to the interface with a zero value at $z^{*}=0$.

In the present case, the existing experimental data do not allow a definitive conclusion about the behaviour of $\overline{\omega^{*} c^{*}}$. Positive and negative values of $\overline{\omega^{*} c^{*}}$ are described in the literature $([5,6,20])$, with both results occurring very close to the surface, being measurement inaccuracies pointed as possible causes of such results. Although frequently presented together with a constant $j^{*}$ profile ( $\omega^{*} c^{*}$ tending to a nonzero constant value far from $\left.z^{*}=0\right)$, data obtained for water in closed tanks do not lead to constant $j^{*}$ along $z^{*}$, even near to the 


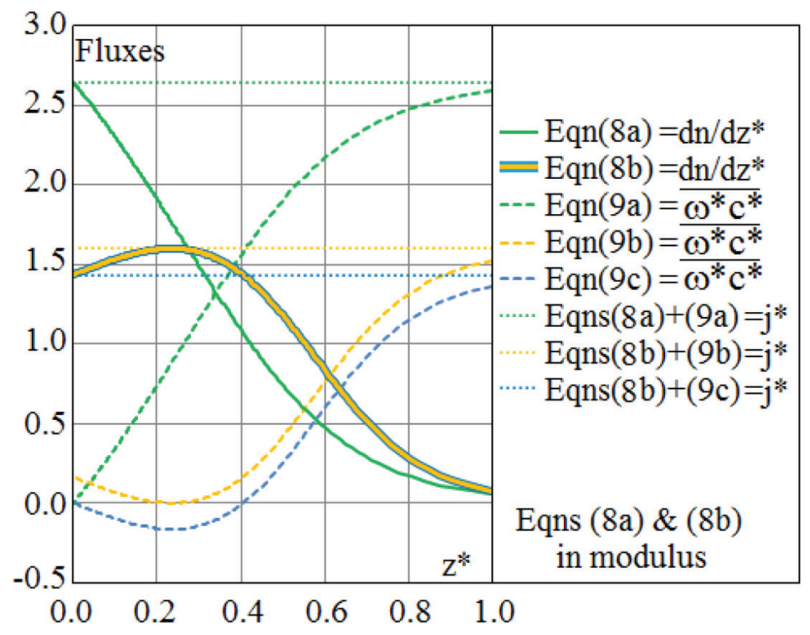

Figure 7: Diffusive flux $d n / d z^{*}$; turbulent flux $\overline{\omega^{*} c^{*}}$, and total constant flux $j^{*}$.

concentration boundary layer. In Ref. [8] the authors discussed this point in their approach for turbulent fluxes using Taylor series around $z^{*}=0$.

Figure 8 shows the envelope of experimental data of $\overline{\omega^{*} c^{*}}$ for several agitation conditions obtained by Ref. [5], together with eqns (9a), (9b) and (9c). The horizontal axis follows the same definition used in Fig. 6. The envelope shows possible positive, null, and negative values of $\overline{\omega^{*} c^{*}}$ close to $z^{*}=0$. The negative range of $\overline{\omega^{*} c^{*}}$ would imply, when using eqn (2), also negative values of the RMS velocity, a situation not observed in the experimental data. The growing region of the experimental data for $z^{+}<\sim 1$ (dark grey) is followed more closely

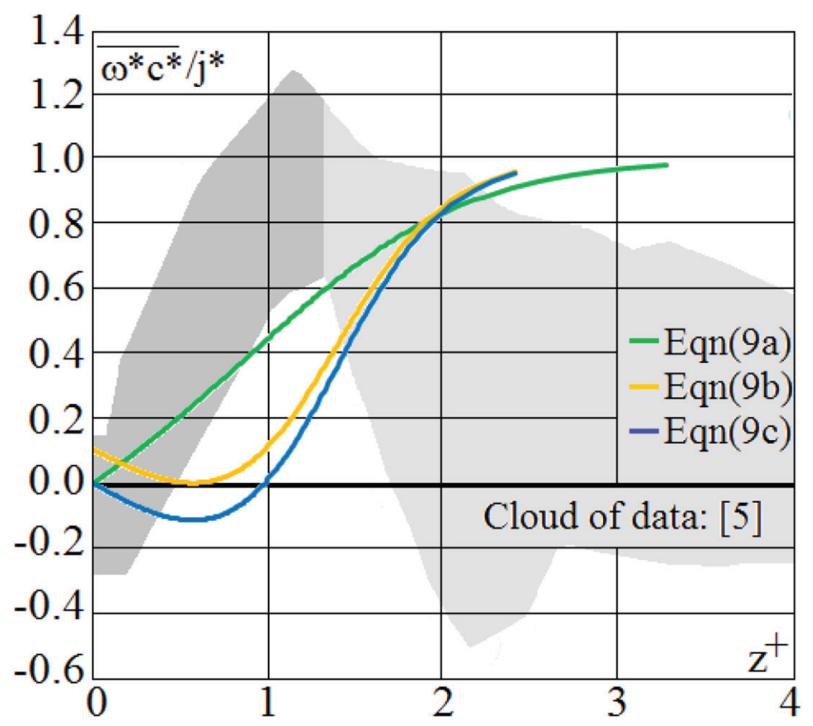

Figure 8: Normalized theoretical turbulent fluxes from indicated equations, and envelope of measured values showing the decreasing of the turbulent flux for $\mathrm{z}^{+}>\sim 1$. 
by eqn (9a), although the slope is better reproduced by eqns (9b) and (9c). The decreasing of the data for $z^{+}>\sim 1$ (light grey) ratifies that the constant flux condition is not attained in closed tanks, even near the interface. The mass accumulates in the closed volume, don't being transported far away. The study of Ref. [8] presents experimental $\overline{\omega^{*} c^{*}}$ results with null values of the functions and their derivatives at $z^{*}=0$, a situation better approached by eqn $(9 \mathrm{~b})$, although its null value is deviated to $z^{*}=0.23$ (or $z^{+}=0.57$ ). As already mentioned, the equations used in this study reflect the constant $\alpha$ condition, which restrict the possibility of further adjustments. Additional studies considering the non-constant $\alpha$ condition are thus desirable.

\subsubsection{The RMS Velocity Function}

Equation (2) allows writing the turbulent intensity (RMS velocity) in the following manner:

$$
\sqrt{\overline{\omega^{* 2}}}=\frac{\overline{\omega^{*} c^{*}}}{n(1-n) A} \sqrt{n(1-n)+\frac{\beta(1-\beta)}{(2 \beta-1)^{2}}}
$$

$\beta$, the superposition function is still unknown. This function assumes the value 1.0 in the RSW method when the records of velocity and concentration fluctuations superpose perfectly [12]. In the present case it implies that a volume of water descending into the bulk liquid carries the concentration of the surface, and a volume of water ascending to the surface carries the concentration of the bulk liquid. This situation is expected to occur close to the interface, so that $\beta=\sim 1$ in this region may be assumed as a first approximation, and eqn (10) simplifies to:

$$
\sqrt{\overline{\omega^{* 2}}}=\frac{\overline{\omega^{*} c^{*}}}{\sqrt{n(1-n) A}}=\frac{\overline{\omega^{*} c^{*}}}{\sqrt{\overline{c^{* 2}}}} \text { or } \overline{\omega^{*} c^{*}}=\sqrt{\overline{\omega^{* 2}}} \sqrt{\overline{c^{* 2}}}
$$

Considering here only the case of $n$ profiles without inflexion points (derivatives without critical points), eqn (11) can be easily calculated because eqns (5a) and (7a) furnish $\sqrt{c^{*^{2}}}$, and eqn (9a) furnishes $\overline{\omega^{*} c^{*}}$. For the mentioned equations, Fig. 9 shows the $\sqrt{\overline{\omega^{* 2}}}$ profile obtained from the RSW method, evidently applied to the space of the concentration boundary layer, together with experimental data from the literature, obtained as nearer as possible to the interface, but not entering the boundary layer. The measured values were obtained from the references [1] and [20]. In order to consider similar experimental conditions of both sources, the data having similar reference turbulence velocities at the surface were used. The reference

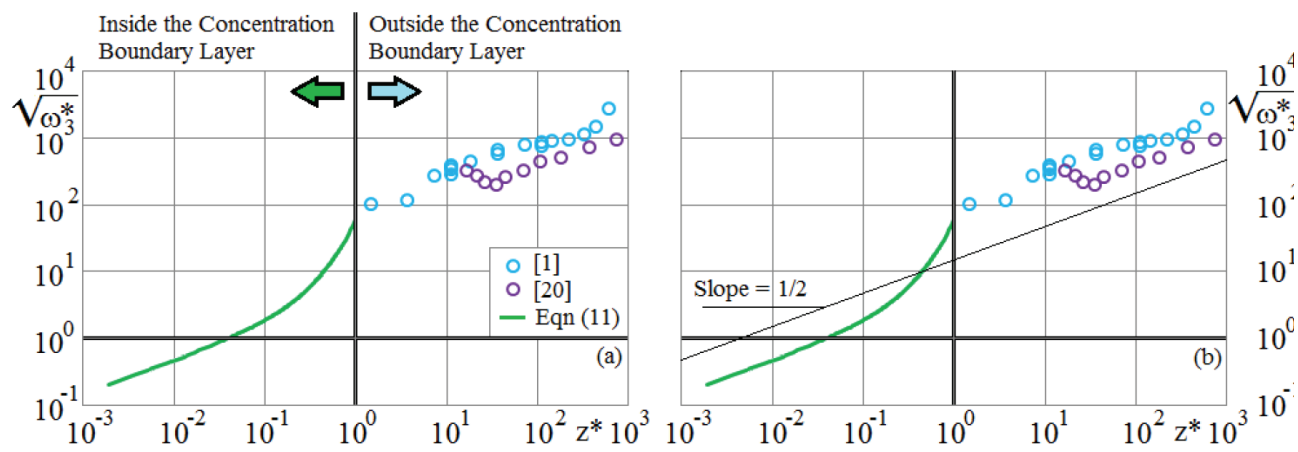

Figure 9: (a) Inner and outer sides of the boundary layer; (b) Overall $1 / 2$ slope trend. 
velocity was the so-called 'Hopfinger and Toly velocity', defined for oscillating grid setups. This velocity scale is given as $7.27 \mathrm{~mm} / \mathrm{s}$ for the data of Ref. [20], and was calculated as 6.83 $\mathrm{mm} / \mathrm{s}$ for the data of Ref. [1]. Because of the proximity of the results, the same mass transfer velocity was used in both cases, with a value of $7.9 .10^{-6} \mathrm{~m} / \mathrm{s}$ for oxygen being absorbed by water, furnished by Ref. [20]. The molecular diffusivity of the oxygen into water was taken as $2 \cdot 1 \cdot 10^{-9} \mathrm{~m}^{2} / \mathrm{s}$. These parameters allowed normalizing the data in order to compare them with the theoretical trends.

Figure 9a describes the condition of this comparison, with the present formulation applied to the inner side of the boundary layer, and the experimental data in the outer side. Fig. $9 \mathrm{~b}$ shows the auxiliary line with slope $1 / 2$, which seems to be followed as an 'overall' trend inside and outside the boundary layer. The study of Ref. [1] compares their data with a 1/3 slope, but in the present analysis the 1/2 trend also composes well with the additional data of Ref. [20]. At the end of the boundary layer the theoretical predictions produce higher slopes.

It is interesting to note that the present evaluations of the RMS velocity are furnished without solving the equation for the movement of the liquid. Equations (10) and (11) follow from the mass conservation equation.

\section{CONCLUSIONS}

The comparisons presented in this study show that, although using the simplifying hypothesis of a constant reduction function $(\alpha)$ in the RSW method, the theoretical behaviour of different statistical functions follow the general shape of measured data. In particular, the mean concentration is very well reproduced by the formulation for $\theta_{1}=2$, a fact already emphasized in previous studies. On the other hand, this simplifying hypothesis leads to differences between experiments and theoretical calculations when considering details close to the interface for the statistical parameters associated to mass transfer. Because the RMS concentration and the turbulent mass flux, two lower order statistical parameters, are more commonly cited in the literature, they were used for the comparisons performed in this study. It was observed that the predictions using $\alpha=0.56091$ (or $\theta_{1}=2$ ) follow better the experimental results of both the RMS fluctuation and the turbulent mass flux. In the last case, the used literature experimental data for turbulent fluxes do not reproduce the condition of constant total flux (the sum of diffusive and turbulent fluxes), because of the accumulation process in the bulk liquid (instead of being transported to greater distances, the mass stays in the closed tank, accumulating). In this case, the solution for $\theta_{1}=2$ followed more adequately the experimental data, however showing a different growing rate of $\overline{\omega^{*} c^{*}}$

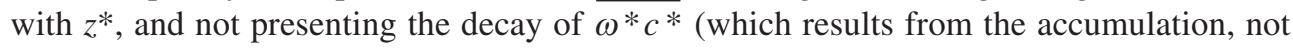
considered in the theoretical solution).

A first evaluation of the RMS velocity was furnished for the near surface region of the concentration boundary layer using the solution for $\theta_{1}=2$. The velocity data used in the present analysis did not attain the concentration boundary layer, but their general trend composed a kind of 'prolongation' of the theoretical solution. Considering the inner and outer sides of the boundary layer, an overall evolution following a $1 \frac{1}{2}$ power law of $z^{*}$ was observed. The result for the RMS velocity was obtained from the formulation for mass conservation in the RSW method. It was not necessary to solve equations for the movement itself.

The best agreement between the experimental data used in this study and theoretical predictions of the different statistical functions (mean concentration, RMS concentration, turbulent flux and RMS velocity) was obtained using the solution for $\theta_{1}=2$. 


\section{ACKNOWLEDGEMENTS}

The first author thanks Brazilian Support Institution CAPES for process BEX 5723/15-9 and Prof. David Zhiwei Zhu for the 2016/17 stay at the University of Alberta, where this study was concluded. The second author thanks CAPES for the fellowship PPG-AEM-EESC-USP.

\section{REFERENCES}

[1] Brumley, B.H. \& Jirka, G.H., Near-surface turbulence in a grid-stirred tank. Journal of Fluid Mechanics, 183, pp. 236-263, 1987. https://doi.org/10.1017/s0022112087002623

[2] Hunt, J.C.R., Turbulent structure and turbulent diffusion near gas-liquid interfaces. Proceedings of the Gas Transfer at Water Surfaces Conference, pp. 67-82, 1984.

[3] Janzen, J.G., Jirka, G.H. \& Schulz, H.E., Details of air-water gas transfer: experiments with precision - in Portuguese. Proceedings of the 16th Brazilian Conference on Water Resources (XVI Simpósio Brasileiro de Recursos Hídricos), pp. 1-17, 2005.

[4] Janzen, J.G., Schulz, H.E. \& Jirka, G.H., Details of air-water gas transfer - in Portuguese. Brazilian Journal of Water Resources, 11(4), pp. 153-161, 2006. https://doi.org/10.21168/rbrh.v11n4.p153-161

[5] Herlina, Gas transfer at the air-water interface in a turbulent flow environment. Dissertation Series of the Institute for Hydromechanics of the University of Karlsruhe, Monograph 2005/4, 2005.

[6] Janzen, J.G., Gas transfer near the air-water interface in oscillating-grid tanks and proprieties of isotropic turbulent flows - in Portuguese. Dissertation at the School of Engineering at São Carlos of the University of São Paulo, 2006.

[7] Janzen, J.G., Herlina, H., Jirka, G.H., Schulz, H.E. \& Gulliver, J.S., Estimation of mas transfer velocity based on measured turbulence parameters. American Institute of Chemical Engineers Journal, 56(8), pp. 2005-2017, 2010. https://doi.org/10.1002/aic.12123

[8] Janzen, J.G., Schulz, H.E. \& Jirka, G.H., Turbulent gas flux measurements near the air-water interface in an oscillating-grid tank. Proceedings of the 2010 Gas Transfer at Water Surfaces Conference, pp. 65-77, 2011.

[9] Herzog, A.G., Imaging of water-sided gas-concentration fields at a wind-driven, wavy air-water interface. Dissertation at the Faculties for the Natural Sciences and for Mathematics Institute of the Ruperto-Carola University of Heidelberg, Heidelberg, Germany, 2010.

[10] Friedl, F., Investigating the transfer of oxygen at the wavy air-water interface under wind-induced turbulence. Dissertation at the Faculties for the Natural Sciences and for Mathematics Institute of the Ruperto-Carola University of Heidelberg, Heidelberg, Germany, 2013.

[11] Krah, N., Visualization of air and water-sided concentration profiles in laboratory gas exchange experiments. Dissertation at the Faculties for the Natural Sciences and for Mathematics Institute of the Ruperto-Carola University of Heidelberg, Heidelberg, Germany, 2014.

[12] Schulz, H.E., Lopes Jr., G.B., Simões, A.L.A. \& Lobosco, R.J., One-dimensional turbulent transfer using random square waves - scalar/velocity and velocity/velocity interactions (Chapter 1). Schulz, H.E., Simões, A.L.A. \& Lobosco, R.J., (eds), Hydrodynamics: Advanced Topics, InTech, Rijeka, pp. 3-34, 2011. 
[13] Schulz, H.E., Simões, A.L.A. \& Janzen, J.G., Statistical approximations in gas-liquid mass transfer. Proceedings of the 2010 Gas Transfer at Water Surfaces Conference, pp. 208-221, 2011.

[14] Schulz, H.E. \& Janzen, J.G., Concentration fields near air-water interfaces during interfacial mass-transport: oxygen transport and random square wave analysis. Brazilian Journal of Chemical Engineering, 26(3), pp. 527-536, 2009. https://doi.org/10.1590/s0104-66322009000300008

[15] Gonçalves, B.B., Detailing the one-dimensional solution for the RSW method considering a constant reduction function applied to the turbulent interfacial scalar transport - text in Portuguese. Dissertation at the School of Engineering at São Carlos of the University of São Paulo, 2014.

[16] Schulz, H.E. \& Gonçalves, B.B., Solutions of scalar mean profiles close to gas-liquid interfaces under turbulent free slip motion. Proceedings of the Eight Conference on Computational Methods in Multiphase Flow, pp. 33-44, 2015, available at: https:// www.researchgate.net/publication/312171886_Errata_to_-_Solutions_of_scalar_ mean_profiles_close_to_gas-liquid_interfaces_under_turbulent_free_slip_motion_-

[17] Gonçalves, B.B. \& Schulz, H.E., One-dimensional turbulent mass transfer at air-water interfaces: details of discontinuities of derivatives using the RSW method. Proceedings of the Seventh Conference on Computational Methods in Multiphase Flow, pp. 365376, 2013.

[18] Falkenroth, A., Visualization of oxygen concentration profiles in the aqueous boundary layer. Dissertation at the Faculties for the Natural Sciences and for Mathematics Institute of the Ruperto-Carola University of Heidelberg, Heidelberg, Germany, 2007.

[19] Atmane, M.A. \& George, J., Gas transfer cross a zero-shear surface: a local approach. Proceedings of the Gas Transfer at Water Surfaces Conference, Geophysical Monograph 127, pp. 255-259, 2002.

[20] Chu, C.R. \& Jirka, G.H., Turbulent gas flux measurements below the air-water interface of a grid-stirred tank. International Journal of Heat and Mass Transfer, 35(8), pp. 1957-1968, 1992.

https://doi.org/10.1016/0017-9310(92)90198-2 